\title{
The equation and the parameter of the spur system
}

\author{
Galina B. Anisimova*, Rahil B. Shatsova
}

Southern Federal University, Rostov-on-Don, Russia; *Corresponding Author: galina@,iubip.ru

Received 22 April 2010; revised 26 May 2010; accepted 30 May 2010.

\begin{abstract}
The regular position of radioloops in the sky and their angular dimensions are described by an equation with a single parameter $2 \pi / \kappa$. Thus, for loops I-IV $\kappa$ takes values 3, 4, 6 and 9 within average random observational errors; and the relative accuracy is only few percent. The parameter form is similar to the wavelength, expressed via the wave number $\kappa$. It is an argument for the hypothesis of wave nature of spurs. Other arguments are considered as well.
\end{abstract}

Keywords: Spurs; Radioloops; Local System; Galaxy; Structure

\section{INTRODUCTION}

The attention to the Galactic shell structures (radioloops, spurs, bubbles, etc), discovered in the middle of the last century, is not growing weak. Each of them is interesting as the largest objects of the Local System. And the totality of these objects is interesting as a background for both nearby and remote objects, which must be taken into account during the investigations. For example, they polarize the microwave radiation, according to WMAP data [1].

Many authors use the schematic spur maps obtained by Landecker and Wielebinsky [2] over $150 \mathrm{MHz}$ continuous radio emission, Berkhuijsen [3] over $820 \mathrm{MHz}$, etc. It is not convenient, if we work in other projections or in other coordinate system, as well as when we define more precisely several various spur parameters, etc. The given here analytical spurs description, removes these problems of applied character. At the same time it gives the new possibilities in the examination of their nature. It helps us to see the harmony in the spur system geometry.

We can describe the geometry of this system by a united equation, having a single parameter. The angular dimensions and the mutual arrangement of their members in the Local System and in the Galaxy depend on the single parameter $2 \pi / k$, where $k$ is the integer number.
It means the discreteness and, moreover, multiplicity. From the other hand, such parameter is similar to the wave length in the wave process, expressed by wave number $k$. It permits to turn to a hypothesis of the density waves in the interpretation of the observations of this system. It is already usual to present the spiral structure of the Galaxy as a system of density waves, having the wave length about several kiloparsecs. Thus it is not difficult to present the part of the Galaxy, such as a Local System, in the same notion, but having the smaller wave length.

\section{THE SPUR EQUATION}

The equation of celestial section we'll obtain from the solution of spherical triangle, having the vertexes $C_{i}\left(\ell_{i}\right.$, $\left.b_{i}\right)$ - the centre of i-spur, $M(\ell, b)$ - the point on the shell and $\Pi\left(b=90^{\circ}\right)$ - the pole of the Galaxy. According to the cosinus theorem

$$
\cos C_{i} M=\sin b_{i} \sin b+\cos b_{i} \cos b \cos \left(\ell-\ell_{i}\right)
$$

The $\mathrm{C}_{\mathrm{i}} \mathrm{M}$ arc is the radius-vector of the shell of the arbitrary shape. In particular $\mathrm{C}_{\mathrm{i}} \mathrm{M}$ is the arc of the large circle, equal to the radius of the rounded spur, or the half of its angular diameter $\rho_{\mathrm{i}}$. Then the spurs equation in the galactic coordinates is:

$$
\cos \left(\ell-\ell_{i}\right)=\left(\cos 1 / 2 \rho_{i}-\sin b_{i} \sin b\right) / \cos b_{i} \cos b
$$

Here $b_{i}-1 / 2 \rho_{i} \leq b \leq b_{i}+1 / 2 \rho_{i}$.

The Eq. 2 over cosinus gives for each $\mathrm{b}$ two $\ell$ values, symmetrical relatively $\ell_{\mathrm{i}}$.

The parameters $\left(\ell_{i}, b_{i}, \rho_{i}\right)$ with the accuracy of several degrees are given in Table 1 over the Berkhuijsen [3] data of continuous radio emission for the spurs I-IV and over C. Heiles [4] for three HI shells. The distance estimations $\mathrm{r}_{\mathrm{i}}$ for the same structures are also given here, but their relative accuracy is much smaller, than for the angular parameters.

The Eq.2 has the canonical character, which means, that it is similar for the other spherical coordinate system - equatorial, local and others.

The Figure $\mathbf{1}$ is obtained according to (2) and Table 1. It is similar to the Berkhuijsen map, but also several sky 
Table 1. The shell parameters over the radio data.

\begin{tabular}{ccccccc}
\hline $\mathrm{i}$ & Shells & $\ell_{\mathrm{i}}$ & $\mathrm{b}_{\mathrm{i}}$ & $\rho_{\mathrm{i}}$ & $\mathrm{r}_{\mathrm{i}}(\mathrm{pc})$ & $\kappa_{\mathrm{i}}$ \\
\hline 1 & I & $329 \pm 1.5$ & $17.5 \pm 3$ & $116 \pm 4$ & $130 \pm 75$ & $3=1 \times 3$ \\
2 & II & $100 \pm 2$ & $-32,5 \pm 3$ & $91 \pm 4$ & $110 \pm 40$ & $4=(1-1 / 4)^{-1} \times 3$ \\
3 & III & $124 \pm 2$ & $15.5 \pm 3$ & $65 \pm 3$ & $150 \pm 50$ & $6=2 \times 3$ \\
4 & IV & $315 \pm 3$ & $48.5 \pm 1$ & $40 \pm 2$ & $250 \pm 90$ & $9=3 \times 3$ \\
5 & Gum Neb & $258 \pm 2$ & $-2 \pm 1$ & 36 & 415 & $10=(1-1 / 10)^{-1} \times 9$ \\
6 & Eridan & 205 & -19 & 40 & 440 & $9=3 \times 3$ \\
7 & Orion Neb & 209 & -19 & 40 & 440 & $9=3 \times 3$ \\
\hline
\end{tabular}

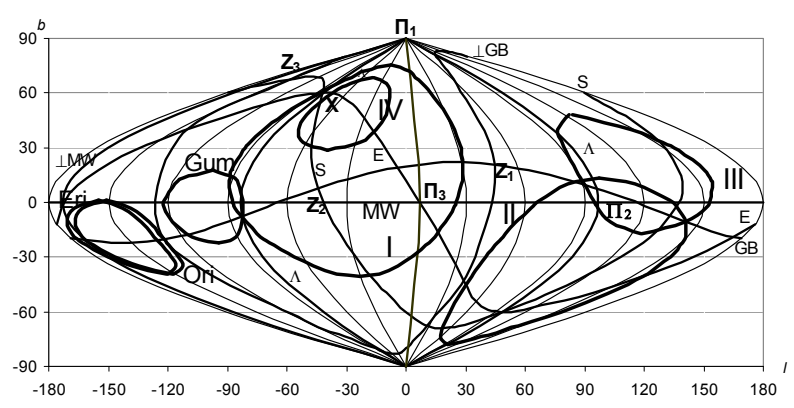

Figure 1. The analytical map of the radioloop system in the galactic coordinates. The circles of local and galactic triedrs and their poles $Z_{1}, Z_{2}, Z_{3}$ and $\Pi_{1}, \Pi_{2}, \Pi_{3}$ are shown.

orienteers, mentioned below, are added.

\section{THE SPURS SYSTEM}

The similarity in the astronomical sense, the spherical shape of large objects and mutual proximity $\left(r_{i} \leq 0,5 \mathrm{kpc}\right)$ permit us to combine all these spurs into the united system (or the subsystem of Local system). Let us join to these features several else, following from the observations and showing the peculiarities of the Eq.2.

\subsection{The Angular Loop Diameters}

The angular loop diameters $\rho_{\mathrm{i}}$ within average random observational errors over the Table 1 data can be rounded up to

$$
\rho_{\mathrm{i}}=2 \pi / \kappa_{\mathrm{i}}
$$

where $\kappa_{\mathrm{i}}$ is the integer number: $3,4,6,9,10$. Moreover, for the majority of the shells these numbers are divisible to 3. To have no exclusions, we may perform (3) for Loop II, as a combination

$$
\rho_{\text {II }}=2 \pi / 4=2 \pi / 1 \times 3-2 \pi / 4 \times 3,
$$

here both members have the denominators, divisible by the typical number $k_{0}=3$. For Gum Neb, having $k=9$ or 10 , the second variant may perform the combination

$$
10=(3+1 / 3) \times 3=(1-1 / 10)^{-1} \times 9 .
$$

The Local bubble also is a part of spurs system [5]. As the Sun is inside this bubble, its $\kappa_{\mathrm{i}}=1$.

If the linear radiuses of the loops from Table 1 are almost the same (about $100 \mathrm{pc}$ ), as admit Berkhuijsen [3] and other authors, then (3) means the dependence for the distances $r$ :

$$
\mathrm{r}_{\mathrm{i}} / \mathrm{r}_{\mathrm{j}}=\left(\sin \rho_{\mathrm{j}} / 2\right) /\left(\sin \rho_{\mathrm{i}} / 2\right) \approx \sin \left(\pi / \kappa_{\mathrm{j}}\right) / \sin \left(\pi / \kappa_{\mathrm{i}}\right)
$$

The $r$ estimations and their large mean square errors do not exclude it. For instance, the nearby Loop I and about 2,5-3 times more remote Gum Neb and Eridan Loop.

\subsection{The Loops Arrangement Relatively the Poles}

We have shown in [6] and [7] that the centers of radioloops I-IV are situated on the small circle $S^{\prime}$, parallel to the equator S (Figure 1), having the common pole $Z_{1}$. The large part of spur areas is arranged at the sky zone between S and S", that had formed its name "the Spurs Belt".

$Z_{1}$ is situated near or even on the intersection of the circles of "the Dolidze net" [8], describing the Local system. This net includes the belts - Gould, VaucouleursDolidze, the perpendicular to them and others, connected with the spurs. The Gould belt (GB) (Figure 1) passes through the nucleuses of Loop I and Eri-Ori complex, through the intersection of Loops II and III, almost touches the Gum Neb. $\perp_{\mathrm{GB}}$ circle divides the spurs system into two groups: (the Loops I, IV, Gum Neb) and (the Loops II, III, Eri-Ori), and it touches the Loop II shell and recently discovered Loop VI [9].

The circles $S, G B$ and $\perp_{\mathrm{GB}}$ form the local triedr $\left(Z_{1}\right.$, $Z_{2}, Z_{3}$ ). The coordinates of triedr poles we can obtain over the coordinates of I-IV centres from Table 1, using (1) for $M=Z_{1}$. The approximate $\ell_{Z 1}$ we can obtain making equal the right parts of 
$\cos Z_{1} C_{i}=\sin b_{z 1} \sin b_{i}+\cos b_{z 1} \cos b_{i} \cos \left(\ell_{i}-\ell_{z 1}\right)$

for Loops I and III and taking into account $\mathrm{b}_{\mathrm{I}} \approx \mathrm{b}_{\mathrm{III}}$. Using Loops II and III and the obtained $\ell_{\mathrm{Z} 1}$, we derive $b_{\mathrm{Z} 1}$. So, in accuracy of several degrees $\ell_{\mathrm{Z} 1}=47^{\circ}, \mathrm{b}_{\mathrm{Z} 1}=21^{\circ}$.

We can obtain $Z_{2}\left(315^{\circ}, 7^{\circ}\right)$ and $Z_{3}\left(207^{\circ}, 68^{\circ}\right)$, using (5) and the circles $S$ and $\perp_{\mathrm{GB}}$ parameters [7] for orthogonal directions of axis of one of the triedrs.

Let us note, that the inclination of $\mathrm{Z}_{3}$ axis and large circle $\mathrm{S}$ to the Galactic plane (MW) is about $2 \pi / 5$, and $\mathrm{b}_{\mathrm{Z} 1} \approx \pi / 2-2 \pi / 5$.

$\mathrm{p}_{1}, \mathrm{p}_{2}$ and $\mathrm{p}_{3}$ - the polar distances of the spur centres $C_{i}$ from the poles of $Z_{1}$ - triedrs are given in the Table 2 for four main spurs confirm the $Z_{1}$ coordinates and its equidistant position, that we obtained firstly in [6] by the other method.

where $\mathrm{p}_{1} \equiv \mathrm{p}_{1}(\mathrm{i})$ (analogous for $\mathrm{p}_{2}$ and $\mathrm{p}_{3}$ )

The concrete $\mathrm{p}_{1}$ differ from the mean $\left\langle\mathrm{p}_{1}\right\rangle=74^{\circ} \approx$ $2 \pi / 5$ within average random observational errors of $\mathrm{C}_{\mathrm{i}}$ coordinates. It is interesting that $\mathrm{p}_{1} \approx 2 \times 2 \pi / 5$ for Gum Neb, and $\mathrm{p}_{1} \approx \pi / 2+2 \pi / 5$ for Eri-Ori complex, that means that each $p_{1}$ corresponds to (3) or their combinations when $\kappa=5$. But here they are related to $Z_{1}$ pole and .radius $\mathrm{Z}_{1} \mathrm{C}_{\mathrm{i}}$, but not the diameter.

Table 2 also shows the interesting combinations of spurs localization relative the other axes of Local triedr $\left(Z_{2}\right.$ and $\left.Z_{3}\right)$, symmetrical relative the opposite poles, similar at the same distances. And the relations between all system members, that is:

$$
\begin{aligned}
& \mathrm{p}_{2,3}(\mathrm{II})+\mathrm{p}_{2,3}(\mathrm{IV}) \approx \pi, \text { in the plane } \mathrm{S} \\
& \mathrm{p}_{3}(\mathrm{I}) \approx \mathrm{p}_{3}(\mathrm{III}) \approx \mathrm{p}_{3}(\mathrm{Gum}) \approx \mathrm{p}_{3}(\mathrm{II})-\mathrm{p}_{3}(\mathrm{IV}) \approx 2 \pi / 5 \\
& \mathrm{p}_{2}(\mathrm{III})-\mathrm{p}_{2}(\mathrm{II}) \approx \mathrm{p}_{2}(\mathrm{Gum})-\mathrm{p}_{2}(\mathrm{IV}) \approx \\
& \mathrm{p}_{2}(\text { Ori })-\pi / 2=\mathrm{p}_{2}(\mathrm{I}) \approx \pi / 2-2 \pi / 5 \\
& \mathrm{p}_{3}(\text { Eri })=\mathrm{p}_{3}(\text { Ori }) \approx \pi / 2, \quad \text { etc }
\end{aligned}
$$

Table 2. The polar distances of loop centres from the triedr poles.

\begin{tabular}{cccccccc}
\hline & & \multicolumn{2}{c}{ Local triedr $Z_{1} Z_{2} Z_{3}$} & \multicolumn{3}{c}{ Galactic triedr $\Pi_{1} \Pi_{2} \Pi_{3}$} \\
\cline { 3 - 8 } i & Loop & $\mathrm{p}_{1}$ & $\mathrm{p}_{2}$ & $\mathrm{p}_{3}$ & $\mathrm{p}_{1}{ }^{{ }^{\prime}}$ & $\mathrm{p}_{2}{ }^{{ }^{\prime}}$ & $\mathrm{p}_{3}{ }^{\prime}$ \\
\hline 1 & $\mathrm{I}$ & 74 & 17 & 75 & 72,5 & 126 & 41 \\
2 & II & 73 & 139 & 126 & 122,5 & 33 & 93 \\
3 & III & 72 & 155 & 73 & 74,5 & 31 & 116 \\
4 & IV & 76 & 41 & 52 & 41,5 & 121 & 66 \\
5 & Gum & 145 & 57 & 78 & 92 & 161 & 109 \\
6 & Eri & 159 & 111 & 87 & 109 & 107 & 154 \\
7 & Ori & 168 & 107 & 87 & 109 & 111 & 151 \\
\hline
\end{tabular}

where $\mathrm{p}_{1} \equiv \mathrm{p}_{1}(\mathrm{i})$ (analogous for $\mathrm{p}_{2}$ and $\left.\mathrm{p}_{3}\right)$
The second part of Table 2 gives the polar distances $\mathrm{p}$ ' from the poles of the Galactic triedr: $\mathrm{p}_{1}$ ' from $\Pi_{1} \equiv \Pi$ $\left(\mathrm{b}=90^{\circ}\right), \mathrm{p}_{2}$ 'from $\Pi_{2} \equiv(\mathrm{MW}, \Lambda)$ at $\left(\ell=97^{\circ}, \mathrm{b}=0\right)$ and $\mathrm{p}_{3}{ }^{\prime}$ from $\Pi_{3} \equiv\left(\mathrm{MW}, \perp_{\mathrm{MW}}\right)$ at $\left(\ell=7^{\circ}, \mathrm{b}=0\right)$, (Figure 1).

Here $\Lambda$ is the Polar Ring of the Galaxy, intersecting MW at the longitudes $\ell=97^{\circ}$ and $277^{\circ}$. Both bright and faint stars, as well as the galaxies of the Local group [10] and the Virgo supergalaxy [11] are concentrated to the $\Lambda$ circle. And relative to the shells: $\Lambda$ touches the loops I, III and Gum, passes through the centre of Loop II. The $\perp_{\mathrm{MW}}$ circle, orthogonal both MW, and $\Lambda$, intersects the most active Loop I region-the North Polar Spur and touches the Eridan shell (Figure 1).

The Loops I and III centres have almost the same $\mathrm{p}_{1}$ $\approx 2 \pi / 5$; the same as $p_{1}$ and $p_{3}$, according to Table 2 . Thus, the Loops I and III centres are equidistant from the northern poles $\Pi_{1}, Z_{1}$ и $Z_{3}$, are symmetrically arranged relative the $\Pi_{1}, Z_{1}$ plane. The triangles $C_{I} \Pi_{1} Z_{1}$ and $C_{\text {III }}$ $\Pi_{1} Z_{1}$ have almost equal sides $\approx 2 \pi / 5$. The Eri and Ori centres are at the same distance from the southern poles $\Pi_{1}, \Pi_{2}$ and $Z_{2}$, and Gum from $\Pi_{3}$. There are interesting combinations also in $\mathrm{p}_{2}{ }^{\prime}$ and $\mathrm{p}_{3}{ }^{\prime}$, as well as between them and $\mathrm{p}_{1}{ }^{\prime}$, including $\kappa=2,3,4,5,12$ :

$$
\begin{aligned}
& \mathrm{p}_{2}{ }^{\prime}(\mathrm{II}) \approx \mathrm{p}_{2}{ }^{\prime}(\mathrm{III}) \approx \mathrm{p}_{2}{ }^{\prime}(\mathrm{I})-\pi / 2 \approx \pi / 6 \\
& \mathrm{p}_{1}{ }^{\prime}(\mathrm{Gum}) \approx \mathrm{p}_{3}{ }^{\prime}(\mathrm{II}) \approx \pi / 2 \\
& \mathrm{p}_{3}{ }^{\prime}(\mathrm{Ori}, \mathrm{Eri}) \approx \pi-\pi / 6 \\
& \mathrm{p}_{2}{ }^{\prime}(\mathrm{I}) \approx \mathrm{p}_{2}{ }^{\prime}(\mathrm{IV}) \approx \mathrm{p}_{1}{ }^{\prime}(\mathrm{II}) \approx 2 \pi / 3 \\
& \mathrm{p}_{2}{ }^{\prime}(\mathrm{Gum}) \approx \pi / 2+2 \pi / 5 \\
& \mathrm{p}_{3}{ }^{\prime}(\mathrm{III}) \approx \pi-\mathrm{p}_{3}{ }^{\prime}(\mathrm{IV})
\end{aligned}
$$

The numerous relations inside each triedr and between them for all shells show the unity and the harmony of this system itself as well as the relations of the triedrs presenting the Galaxy and Local system.

\subsection{The Mutual Spurs Arrangement}

The position of Loops I-IV centres on $\mathrm{S}^{\prime}$ circle $\left(\mathrm{p}_{1} \approx\right.$ $2 \pi / 5)$ is not random. One can see this from (6) и (7). The differences of the azimuthal coordinates $\Delta \mathrm{s}$ in the Local System (s, p) show this more concentrative. The angle $s$ can be obtained from the triangle $Z_{1} \Pi_{1} C_{i}$

$$
\sin \mathrm{p}_{1} \sin \left(\mathrm{s}-\mathrm{s}_{\Pi}\right)=\operatorname{cosb} \sin \left(\ell-\ell_{\mathrm{Z} 1}\right)
$$

here $s_{\Pi}$ is the angle for the northern pole of the Galaxy. If $s$ begins from the Loop I centre $\left(\mathrm{s}_{\mathrm{I}}=0\right)$, then $\mathrm{s}_{\Pi}=$ $285^{\circ}$.

The angular distances between the centres of the neighbouring loops can be rounded in each of two groups up to

$$
\begin{aligned}
& \mathrm{S}_{\mathrm{III}}-\mathrm{S}_{\mathrm{II}}=56^{\circ} \approx 2 \pi / 6=\pi / 2-\pi / 6 \\
& \mathrm{~S}_{\mathrm{I}}-\mathrm{S}_{\mathrm{IV}}=34^{\circ} \approx \pi / 6
\end{aligned}
$$


the last one is the maximal relative deviations from (3). Between the members of the opposite groups:

$$
\begin{aligned}
& \mathrm{S}_{\mathrm{II}}-\mathrm{S}_{\mathrm{I}}=149^{\circ} \approx \pi-\pi / 6 \text { and } \\
& \mathrm{S}_{\mathrm{IV}}-\mathrm{S}_{\mathrm{III}}=121^{\circ} \approx 2 \pi / 3=\pi-2 \pi / 6=\pi / 2+\pi / 6,
\end{aligned}
$$

it means,

$$
\mathrm{S}_{\mathrm{II}}-\mathrm{S}_{\mathrm{IV}}=177^{\circ} \approx \pi ; \quad \mathrm{S}_{\mathrm{III}}+\mathrm{s}_{\mathrm{IV}} \approx \pi
$$

That is, the centres of II and IV are in opposition at

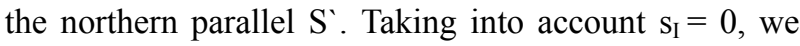
have the rounded coordinates:

$$
\mathrm{s}_{\mathrm{II}}=\pi-\pi / 6, \mathrm{~s}_{\mathrm{III}}=\pi+\pi / 6, \mathrm{~s}_{\mathrm{IV}}=-\pi / 6 .
$$

The HI shells are situated at the southern parallels: $\mathrm{S}^{\text {" }}$ at $\mathrm{p}_{1}=2 \times 2 \pi / 5$ and $\mathrm{S}^{\prime \prime}$ at $\mathrm{p}_{1}=\pi / 2+2 \pi / 5$ (Table 2). Their s-coordinates are related:

$$
\begin{aligned}
& \mathrm{s}(\text { Ori, Eri) }-\mathrm{s}(\mathrm{Gum}) \approx \pi-\pi / 6, \\
& \mathrm{s}(\text { Ori, Eri) }+\mathrm{s}(\mathrm{Gum}) \approx \pi
\end{aligned}
$$

Thus, all three parameters $\left(\mathrm{s}_{\mathrm{i}}, \mathrm{p}_{\mathrm{i}}, \rho_{\mathrm{i}}\right)$ of the equation of (2) type are given approximately in (3) shape, or their combination, being the functions of $\kappa$ numbers, having the typical values 6,5 and 3 .

The Table 3 gives these roundnesses $\left(\mathrm{s}_{\mathrm{i}}{ }^{\circ}, \mathrm{p}_{1}{ }^{\circ}, \rho_{\mathrm{i}}{ }^{\circ}\right)$ and the galactic coordinates of centres $\left(\ell_{i}^{\circ}, b_{i}{ }^{\circ}\right)$, obtained over the transference formulas. They differ from the Table 1 $\left(\ell_{\mathrm{i}}, \mathrm{b}_{\mathrm{i}}\right)$ values within average random observational errors, or of angular shells' thickness. That is why Figure $\mathbf{1}$ is obtained, using (2) and Table $\mathbf{3}$, as well as Table $\mathbf{1 .}$ It is similar to the Berkhuijsen map [3].

Here $\mathrm{p}_{1}^{\circ} \equiv \mathrm{p}_{1}{ }^{\circ}(\mathrm{i})$.

One can write the equation of (2) type for main four spurs, but using $(\mathrm{s}, \mathrm{p})$ coordinates on the shells:

$$
\cos \left(\mathrm{s}-\mathrm{f}_{\mathrm{i}}(\pi / 6)\right)=\frac{\left[\cos \pi / \kappa_{\mathrm{i}}-\cos p \cos 2 \pi / 5\right]}{\sin p \sin 2 \pi / 5}
$$

so far as $C_{i}\left(s_{i}{ }^{\circ}=f_{i}(\pi / 6), p_{i}=2 \pi / 5\right)$ is the centre of $i$-shell, and $\mathrm{s}_{\mathrm{I}}=0$.

(13) has the single parameter $\kappa_{i}$ or in the parametric form:

$$
\mathrm{s}_{\mathrm{i}}=\mathrm{s}_{\mathrm{i}}\left(\kappa_{\mathrm{i}}\right), \mathrm{p}_{\mathrm{i}}=\mathrm{p}_{\mathrm{i}}\left(\kappa_{\mathrm{i}}\right), \rho_{\mathrm{i}}=\rho_{\mathrm{i}}\left(\kappa_{\mathrm{i}}\right)
$$

As an example we can see the s coordinates for points

Table 3. The parameters of spur equations in approach of $2 \pi / \kappa$.

\begin{tabular}{ccccccc}
\hline $\mathrm{i}$ & Loop & $\mathrm{s}_{\mathrm{i}}-\mathrm{s}_{\mathrm{I}} \approx \mathrm{f}_{\mathrm{i}}(\pi / 6)$ & $\mathrm{p}_{1}$ & $\rho_{\mathrm{i}}$ & $\ell_{\mathrm{i}}$ & $\mathrm{b}_{\mathrm{i}}$ \\
\hline 2 & $\mathrm{I}$ & 0 & $2 \pi / 5$ & $2 \pi / 3$ & $330^{\circ}$ & $20^{\circ}$ \\
3 & II & $\pi-\pi / 6$ & $2 \pi / 5$ & $2 \pi / 4$ & 100 & $-33,5$ \\
4 & III & $\pi+\pi / 6$ & $2 \pi / 5$ & $2 \pi / 6$ & 124 & 18,6 \\
5 & IV & $0-\pi / 6$ & $2 \pi / 5$ & $2 \pi / 9$ & 311 & 48,3 \\
6 & Gum & $1 / 2 \times \pi / 6$ & $2 \times 2 \pi / 5$ & $2 \pi / 9$ & 258 & 2 \\
7 & Ori & $\pi-1 / 2 \pi / 6$ & $2 \pi / 4+2 \pi / 5$ & $2 \pi / 9$ & 210 & -21 \\
\hline
\end{tabular}

of the intersection of the four loops with the S circle, having $\mathrm{p}=90^{\circ}$ and

$$
\cos \left(\mathrm{s}_{\mathrm{i}}^{\circ}-\mathrm{s}\right)=\frac{\cos \pi / \kappa_{\mathrm{i}}}{\sin 2 \pi / 5}=\frac{\sin \left(\pi / 2-\pi / \kappa_{\mathrm{i}}\right)}{\sin 2 \pi / 5}
$$

when $\kappa_{i}=3,4,6,9$.

\section{THE LENS II-III}

The arrangement of "the Lens" in the intersection of II and III shells is one more improvement of internal and external relations. The Lens is turned to us by its edge and it is stretched for $\approx 57^{\circ} \approx \pi / 3$ along the Gould Belt. The Lens centre coincides the node (GB, MW). The Lens vertexes $\mathrm{A}\left(\ell \approx 143^{\circ}, \mathrm{b}=-11^{\circ}\right)$ and $\mathrm{B}\left(91^{\circ},+12^{\circ}\right)$ have the antipodes in Loop I nucleus and on its shell near Gum Neb. At the same time the B vertex is situated near the quadratures, with the centre and the anticentre of the Galaxy $(\kappa=4)$. We paid our attention in [12] to the fact that many interesting sky objects are observed through this Lens: they are the bright part of Perseus arm together with its OB associations and 5 of 12 historical supernova, etc. It makes this sky region rather famous.

$$
\text { - " - }
$$

The equations of (3) type are typical, when $\kappa$ are integer in all examined positions. This property may formally be grounded by small relative error $\Delta \kappa / \kappa$ both in $\Delta \rho / \rho, \Delta \mathrm{p} / \mathrm{p}$ and $\Delta \mathrm{s} / \mathrm{s} . \rho$ and $\Delta \rho$ are the values taken from Table 1. $\Delta \mathrm{p}$ and $\Delta \mathrm{s}$ are the differences between the real and mean values or between the calculated and rounded ones, over Table 2 and 3. They show the observation accuracy about several degrees. As a result, the mean relative error of $\kappa$ is about $4 \%$. The maximal deviation in (9) is $34^{\circ}-\pi / 6=4^{\circ}$ or $4 / 30$, that is $13 \%$. It is more difficult to distinguish the discreteness from the continuality for large $k$.

Let's point out, that we also analyzed [7] the loops attachment to the elements of Z-triedr, and to the "Dolidze net" [8] on the whole. Then the main attention was paid to the regularity, having the period $\pi / \mathrm{n}$ for the net, describing the stellar distribution. Now we showed that the same periodicity of (3) type is inherent also to the spur system.

The number of examples of the regularities outside the Solar system increases, thus, Elmegreen and Elmegreen [13] turned their attention to the regularity in localization of stellar-gas complexes in the spiral arms of many galaxies.

Efremov [14] explains the regularity in the complexes localization in our Galaxy and in Andromeda by the regularity of magnetic fields along their arms.

The concentrations of OB-stars and their associations over Lindblad [15] to the nodes in Figure 2 are evident. 


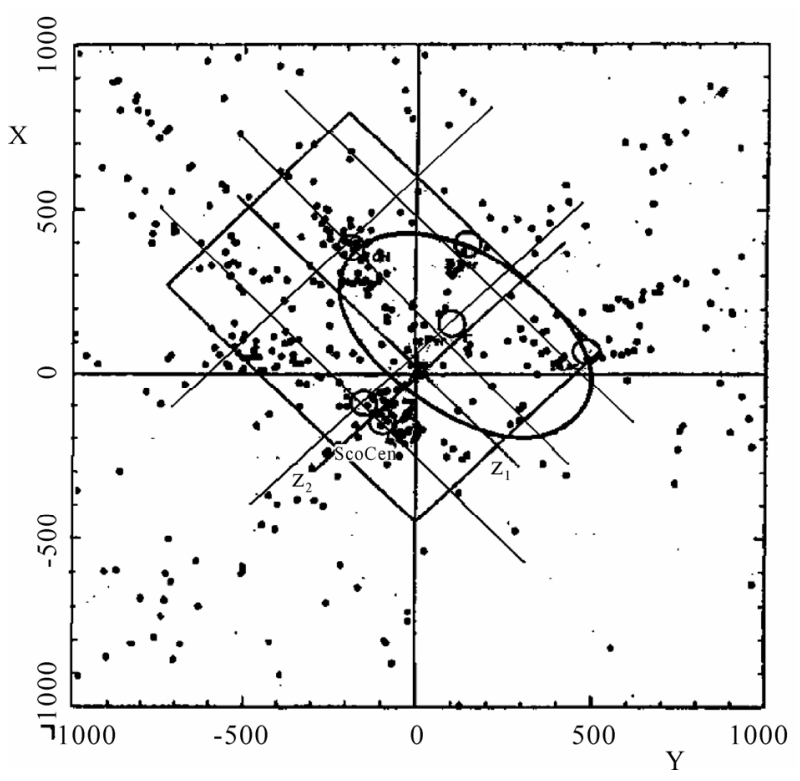

Figure 2. The Lindblad Ring over the [15], the local triedr axis $\mathrm{Z}_{1}$ and $\mathrm{Z}_{2}$ and the ridges net of probable density waves.

Loktin and Popova [16] showed the regular distribution of young open clusters, Cepheids and HII regions and their alternation with older stellar complexes along the spiral arms.

The coincidence of (3) and expression of the wave length through the wave number $\kappa$ may be either formal or it reflects very important property in both the stellar and spur distributions and perhaps the other objects.

\section{FROM THE CELESTIAL PROJECTION TO THE SPACE VIEW}

We examined in [17] the spurs localization in Local System and in the Lindblad Ring over the neutral hydrogen. Continuing this analysis, let's see the correspondence between the elements of Z-triedr and the Ring, according to Lindblad version [15], Figure 2.

$Z_{1}$ axis, passing through the Sun, is parallel to the large Ring axis. $Z_{2}$ axis, lying in the intersection of GB and $\mathrm{S}$, is parallel to its small axis. So the $\mathrm{Z}_{1} \mathrm{Z}_{2}$ triedr plane is parallel to the symmetry plane of the Ring, or to the Gould Belt. The third axis $Z_{3}$, in the intersection of $\perp \mathrm{GB}$ and $\mathrm{S}$, is perpendicular to GB and to the Ring. It is inclined to the Galactic plane (MW) for the angle $\approx 2 \pi / 5$. $\perp$ GB plane, as we mentioned before, divides the spurs system into two groups, lying to both sides from the main $Z_{1}$ axis. I and IV spurs at one side, II and III, at another, and the Local Bubble [5], squeezed between them, are stretched along $S$ ' perpendicularly to $Z_{1}$. They form the united ridge SS'.

The spurs belt $\mathrm{S}$ one can plot parallel to the ridge of ring-similar structures, including Great Rift, passing nearer to $Z_{1}$, that is at the smaller $p_{1}$. It is shown in Figure 3 in the Ring, given by Elmegreen and then reproduced by [18]. The ridge, consisting of the filaments and arcs of rings, including Ori OB1, is arranged at the other side of large axis of the Ring at $p_{1}>\pi / 2$. The whole Ori-Eri complex and, perhaps, Gum Neb, several small $\mathrm{Hu}$ shells [19] etc, are belonging to it. Three or four parallel each other ridges together with the gaps between them form the system of ridges and cavities of density waves, perpendicular to the large axis of the Ring and $Z_{1}$. The wave length is $200-400$ pc. If to take into consideration only I-IV loops, then the small Ring axis is $<500 \mathrm{pc}$. But the last year discoveries can rather move apart the borders of Lindblad Ring. The IRAS loops are discovered in both directions of Spur Belt S up to the Perseus and Sagittarius, as well as in the arms themselves [20]. Behind the Ori-Eri complex, almost in the same direction the Mon R2 ring is stretched out at $\mathrm{r} \approx 830$ pc [4]. The Aquila Rift is in the opposite direction.

Thus, both inside the Lindblad Ring and outside it, we see the density wave net. The regularity along the spiral arms, noted in [13] and [14], perhaps is also connected with this net. The orthogonal ridges differ from the galactic pattern in the wave length about an order smaller and the inclination of main plane. Perhaps, they differs each other as transversal, along $Z_{1}$, and longitudinal waves along the continuous, though inhomogeneous "pivot" S. The loops at this S are coming into contact or are intersecting each one with its own source, from which its own waves are spreading out. Perhaps, this may be the interpretation of the discovered not long ago $\mathrm{V}$ and VI loops [9]. They have the centres near Loop III centre but larger $\rho_{\mathrm{i}}\left(\approx>120^{\circ}\right)$, covering a part of Loop II from one side, and touching Loop I from another.

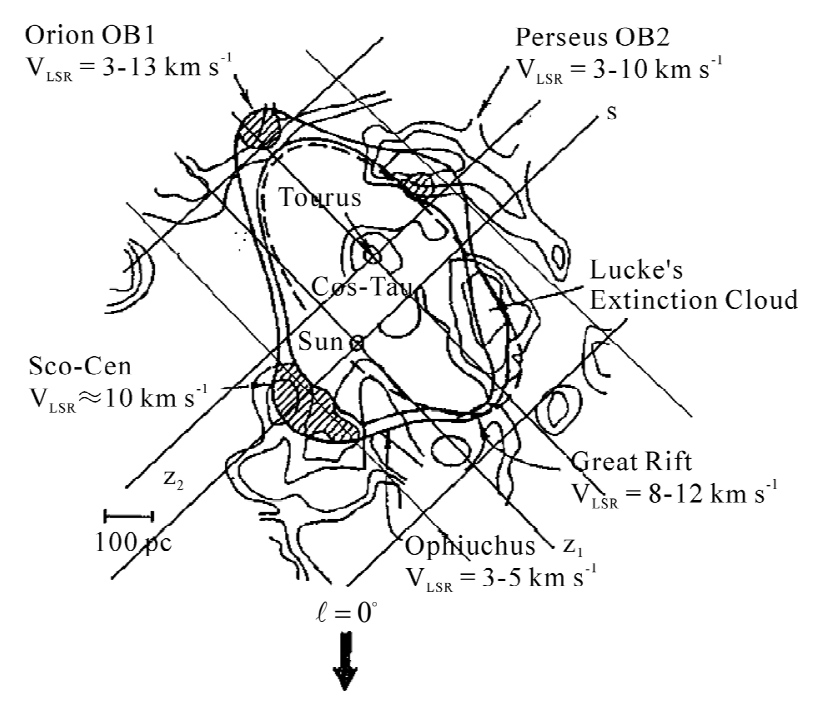

Figure 3. The Lindblad Ring over the Comeron version [18], the axis $Z_{1}$ and $Z_{2}$; and the net ridges of probable density waves. 
And the kinematic aspect must be examined, as well. We paid attention in [6] to the coincidence of the coordinates of Solar motion apex and the Pole of Spur system $Z_{1}$. The basic motion apex $\left(\ell_{\mathrm{A}}=45^{\circ}, \mathrm{b}_{\mathrm{A}}=24^{\circ}\right)$ coincides with $Z_{1}$ in accuracy of several degrees. And (3) takes place relatively the Pole $Z_{1}$ isn't it? The Sun moves parallel to the large axis of the Lindblad Ring, that is along the spreading of possible transversal waves. The same is preferential motion of the majority of stars and moving clusters in the Solar vicinity [21]. The Kovalsky-Kapteyn figures show the same [6].

We think that these facts themselves show the existence of the wave process.

\section{CONCLUSIONS}

The map of spurs system (Figure 1), obtained by the analytic method, is identical to the Berkhuijsen map, obtained over the radio isophots. The spurs equation has 3 parameters: the angular diameters and their centre coordinates. The spurs system is so harmonious, that assume a number of empiric correlations between the elements of all its members. Both the parameters of each spur and the spur system in approach can be described by the single parameter of $2 \pi / \kappa$ type, where $\kappa$ - the integer numbers, many of them are divisible to $\kappa_{0}=3$. The radioloops are the largest objects of Local System. But the statistics is small, as they are not numerous. So, the individual observed data and their accuracy are so important. If the discreteness and multiplicity of $2 \pi / \kappa$ parameter is considered as a hypothesis, then it has very good accuracy (several percents), in comparison to many other astronomical hypotheses.

1) The equation, describing the spurs system geometry, and the analysis of its parameters gives the possibility to see its structure and regular nature from the other side. It permits to create new perspective hypotheses.

The simplified one-parametrical model may be a stage, preceding and approaching the creation of theory of structure - dynamics - evolution of spurs system as a part of the Local System. The harmony of this system shows the united and non-random formation process (instead of, for example, the incidental supernova burst). The type of $2 \pi / \kappa$ parameter (if it is not formal) may be a hint to the wave nature of spur system.

2) Perhaps, the spur groups form the ridges, orthogonal to the spiral arms, and together they form the wave dense net. Thus one ridge $\mathrm{S}$ contains the main spurs I-IV, Local Bubble and several IRAS loops. The other ridges contain the Ori-Eri complex and Gum Neb. But these and several other arguments can not immediately solve the net problem.

3) The analytical approach can be useful in finding new members of spur system and in solving other problems.

\section{REFERENCES}

[1] Page, L., Hinshaw, G., Komatsu, E., et al. (2007) Three year wilkinson microwave anisotropy probe observations: Polarization analysis. The Astrophysical Journal Supplement Series, 170(2), 335-376.

[2] Landecker, T.L. and Wielebinski, R. (1970) The galactic metre wave radiation. Australian Journal of Physics. Astrophysical Supplement, 16, 1-20.

[3] Berkhuijsen, E.M. (1973) Galactic continuum loops and the diameter-surface brightness relation for supernova remnants. Astronomy \& Astrophysics, 24, 143-147.

[4] Heiles, C. (1998) Whence the local bubble, gum, orion? Astrophysical Journal, 498, 689-703.

[5] Lallement, R., Welsh, B.Y., Vergely J.L., et al. (2003) 3D mapping of the dense interstellar gas around the local bubble. Astronomy \& Astrophysics, 411(3), 447-464.

[6] Shatsova, R.B. and Anisimova, G.B. (2002) The spur system in ecliptic coordinates. Astrophysics, 45(4), 535-546.

[7] Shatsova, R.B. and Anisimova, G.B. (2003) The local system harmony. Astrophysics, 46(2), 319-329.

[8] Dolidze, M.V. (1980) Peculiarities of local spiral arm features of the Galaxy. Letters to Astronomical Journal, 6, 745-749

[9] Borka, V., Milogradov-Turin, J. and Uroševic, D. (2008) The brightness of the galactic radio loops at $1420 \mathrm{MHz}$ : some indications for the existence of loops V and VI. Astronomische Nachrichten, 329(4), 397-402.

[10] Drosdovsky, I. (2007) The local group of galaxies. http://www.astronet.ru/db/msg/1169715

[11] De Vaucouleurs, G., Val, A. and Corwin, H.G. (1976) Second reference catalogue of bright galaxies, Austin, London.

[12] Shatsova, R.B. and Anisimova, G.B. (2008) The large sky Lens. Odessa Astronomical Publications, 21, 106-107.

[13] Elmegreen, B.G. and Elmegreen, D.M. (1983) Monthly notices of the royal astronomical society, 203, 31 .

[14] Efremov, Y.N. (2004) Stellar complexes and starburst cumps. Proceedings of Astrophysics and Cosmology after Gamow, Odessa, 90.

[15] Lindblad, P.O. (2000) On the rotation of Gould Belt. Astronomy \& Astrophysics, 363(1), 154-158.

[16] Loktin, A.V. and Popova M.E. (2007) The vavelet-smoothed distribution of young stellar objects in the galactic plane. Astronomicheskǐ Zhurnal, in Russian, 84(5), 409-417.

[17] Shatsova, R.B. and Anisimova, G.B. (1997) The dynamical scheme of local system. In: Agekian, T.A., Mullari, A.A. and Orlov, V.V., Eds., Structure and Evolution of Stellar Systems, St. Peterburg University Press, 184-188.

[18] Comeron, F. (1997) The Gould Belt. In: Agekian, T.A., Mullari, A.A. and Orlov, V.V., Eds., Structure and Evolution of Stellar Systems, St. Peterburg University Press, 161-173.

[19] $\mathrm{Hu}$, E. (1981) High latitude HI shells in the Galaxy.I. Astrophysical Journal, 248, 119-127. 
[20] Könyvers, V., Kiss, Cs., Moor, A., et al. (2007) Catalogue of far-infra-red loops in the Galaxy. Astronomy and Astrophysics, 463(3), 1227-1234.

[21] Popovic, G.M., Ninkovic, S. and Pavlovic, R. (1997) A kinematical approach to the separation of moving-cluster members. In: Agekian, T.A., Mullari, A.A. and Orlov, V.V., Eds., Structure and Evolution of Stellar Systems, St. Peterburg University Press, 108-113. 